\title{
The optical gravitational lensing experiment. Variable stars in globular clusters
}

\section{Fields 5139D-F in $\omega$ Centauri $^{\star}$}

\author{
J. Kaluzny ${ }^{1}$, M. Kubiak ${ }^{1}$, M. Szymański ${ }^{1}$, A. Udalski ${ }^{1}$,W. Krzemiński ${ }^{2}$, M. Mateo ${ }^{3}$, and \\ K. Stanek $^{4}$ \\ 1 Warsaw University Observatory, Al. Ujazdowskie 4, 00-478 Warsaw, Poland \\ e-mail: (jka,mk,msz,udalski)@sirius.astrouw.edu.pl \\ 2 Carnegie Observatories, Las Campanas Observatory, Casilla 601, La Serena, Chile \\ e-mail: wojtek@roses.ctio.noao.edu \\ 3 Department of Astronomy, University of Michigan, 821 Dennison Bldg., Ann Arbor, MI 48109-1090, U.S.A. \\ e-mail: mateo@astro.lsa.umich.edu \\ 4 Princeton University Observatory, Peyton Hall, Princeton NJ 08544, U.S.A. \\ e-mail: stanek@astro.princeton.edu
}

Received April 11; accepted July 23, 1996

\begin{abstract}
Three fields located close to the center of the globular cluster $\omega$ Cen were surveyed in a search for variable stars. We present $V$-band light curves for 32 periodic variables. This sample includes $10 \mathrm{SX}$ Phe stars, 19 eclipsing binaries, and three likely spotted variables (FK Com or RS CVn type stars). Only 5 of these variables were previously known (including two objects reported in Paper I). All SX Phe stars and 8 eclipsing binaries from our sample belong to blue stragglers. Five binaries occupy positions on the upper main-sequence of the cluster and another four are located at the base of the subgiant branch. Of particular interest is detection of a detached binary system with a period $P=4.64$ day. Further study of this star can provide direct information about properties of turnoff stars in $\omega$ Cen. All SX Phe stars and most of eclipsing binaries discovered in our survey are likely cluster members. We present $V$ vs. $V-I$ color-magnitude diagrams for the monitored fields. A populous group of likely hot subdwarfs as well as numerous candidates for blue stragglers can be noted in these diagrams. Our data indicate that $\omega$ Cen possesses the largest number of blue stragglers among all galactic globulars.
\end{abstract}

Key words: globular clusters: individual: $\omega$ Cen — star: variables: other — blue stragglers — binaries: eclipsing HR diagram

Send offprint requests to: J. Kaluzny

* Based on observations collected at the Las Campanas Observatory of the Carnegie Institution of Washington.

\section{Introduction}

The Optical Gravitational Lensing Experiment (OGLE) is a long term project with the main goal of searching for dark matter in our Galaxy by identifying microlensing events toward the galactic bulge (Udalski et al. 1992, 1994). At times the Bulge is unobservable we conduct other long-term photometric programs. A complete list of side-projects attempted by the OGLE team can be found in Paczyński et al. (1995). In particular, during last three observing seasons we monitored globular clusters NGC 104 (= 47 Tuc) and NGC 5139 (= $\omega$ Cen) in a search for variable stars of various types. Of primary interest was detection of detached eclipsing binaries. In Paper I (Kaluzny et al. 1996) we presented results for three fields in $\omega$ Cen. Here we report on variables discovered in another three fields of that cluster.

\section{Observations and data reduction}

The OGLE project is conducted using the 1-m Swope telescope at Las Campanas Observatory which is operated by Carnegie Institution of Washington. A single $2048 \times 2048$ pixels Loral CCD chip, giving the scale of $0.435 \mathrm{arcsec} / \mathrm{pixel}$ is used as the detector. The initial processing of the raw frames is done automatically in nearreal time. Details of the standard OGLE processing techniques are described by Udalski et al. (1992).

This paper is based mostly on the data obtained during 1995 observing season. The cluster was monitored for a period spanning about 3 months. Detailed logs of 
Table 1. Equatorial coordinates for centers of 5139D-F fields

\begin{tabular}{lll}
\hline Field & $\alpha_{2000}$ & $\delta_{2000}$ \\
\hline $5139 \mathrm{D}$ & $13: 25: 21.9$ & $-47: 28: 50$ \\
$5139 \mathrm{E}$ & $13: 28: 02.0$ & $-47: 28: 52$ \\
$5139 \mathrm{~F}$ & $13: 28: 02.3$ & $-47: 40: 57$ \\
\hline
\end{tabular}

Table 2. Number of frames reduced for the $\omega$ Cen fields

\begin{tabular}{lll}
\hline Field & Filter $V$ & Filter $I$ \\
\hline $5139 \mathrm{D}$ & 233 & 36 \\
$5139 \mathrm{E}$ & 282 & 44 \\
$5139 \mathrm{~F}$ & 139 & 10 \\
\hline \hline
\end{tabular}

observations can be found in Udalski et al. $(1995,1996)$. We monitored three fields. Fields 5139D and 5139E were centered about 13 arcmin West and about 13 arcmin East of the cluster center, respectively. Field $5139 \mathrm{~F}$ was centered about 12 arcmin South in respect to field 5139E. The equatorial coordinates of centers of fields 5139D-F are given in Table 1. Fields 5139E and 5139F were observed only in 1995. Field 5139D was additionally monitored for about 5 hours on the night of 21 June, 1995. A schematic chart with marked location of all monitored fields (Papers I and II) is shown in Fig. 1. Most of the monitoring was performed through the Johnson $V$ filter. Some exposures in the Kron-Cousins $I$ band were also obtained. Table 2 gives total numbers of frames reduced for each of surveyed fields. Most of observations in the $V$-band were collected with an exposure time set to $420 \mathrm{~s}$. For the $I$-band exposures lasted $300 \mathrm{~s}$. For majority of the analyzed frames seeing was better than 1.6 arcsec. The reduction techniques as well as algorithms used for selecting potential variables are described in Paper I. The profile photometry was extracted with the help of DoPHOT (Schechter et al. 1993). The total number of stars contained in data bases with $V$ photometry was 37771,49426 and 22115 for fields, 5139D, $5139 \mathrm{E}$ and $5139 \mathrm{~F}$, respectively. Most of these stars were faint objects with relatively large errors of photometry. Table 3 gives condensed information about the numbers of stars analyzed for variability and about the quality of derived photometry.

\section{Variable stars}

In this paper we present results for all variables identified in observed fields with the exception of RR Lyr stars. RR Lyr stars in $\omega$ Cen shall be subject of a separate contribution. We identified a total of 32 periodic variables of which 27 are new discoveries. The rectangular and equatorial coordinates of these stars are listed in Table 4 . The rectan-

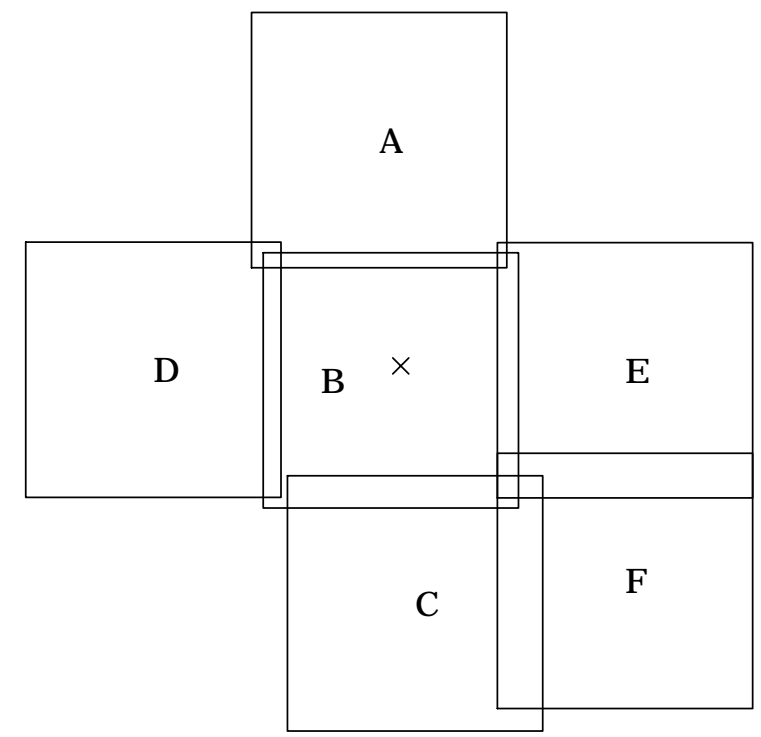

Fig. 1. A schematic chart showing location of fields 5139A-F. The cluster center is marked with cross. Each field covers $14.7 \times 14.7 \mathrm{armin}^{2}$

Table 3. Basic statistical data about stars from fields 5139D-F examined for variability. The data are given for bins $0.5 \mathrm{mag}$ wide. Columns 2, 4 and 6 give median value of rms for a given bin. Columns 3, 5 and 7 give numbers of stars examined for variability

\begin{tabular}{|c|c|c|c|c|c|c|}
\hline \multicolumn{3}{|c|}{ D } & \multicolumn{2}{|l|}{$\mathrm{E}$} & \multicolumn{2}{|l|}{$\mathrm{F}$} \\
\hline$V$ & $<\mathrm{rms}>$ & $N$ & $<\mathrm{rms}>$ & $N$ & $<\mathrm{rms}>$ & $N$ \\
\hline 14.5 & 0.015 & 125 & 0.019 & 175 & 0.014 & 66 \\
\hline 15.0 & 0.010 & 187 & 0.012 & 231 & 0.011 & 107 \\
\hline 15.5 & 0.010 & 144 & 0.013 & 219 & 0.011 & 92 \\
\hline 16.0 & 0.011 & 215 & 0.014 & 270 & 0.012 & 129 \\
\hline 16.5 & 0.013 & 305 & 0.018 & 418 & 0.015 & 152 \\
\hline 17.0 & 0.017 & 392 & 0.021 & 579 & 0.019 & 238 \\
\hline 17.5 & 0.020 & 923 & 0.027 & 1340 & 0.023 & 547 \\
\hline 18.0 & 0.026 & 2129 & 0.036 & 3040 & 0.029 & 1115 \\
\hline 18.5 & 0.036 & 3451 & 0.047 & 5185 & 0.041 & 1913 \\
\hline 19.0 & 0.043 & 1540 & 0.059 & 2200 & 0.056 & 2550 \\
\hline
\end{tabular}

gular coordinates correspond to positions of variables on the $V$-band "template" images which were submitted to the editors of A\&A (see Appendix A). These images allow easy identification of all objects listed in Table 4 . The name of field in which a given variable can be identified is given in the 6th column. All frames collected by the OGLE team were deposited at the NASA NSS Data Center ${ }^{1}$. Frames mr10525, mr10524 and mr11042 were used as

1 The OGLE data (FITS images) are accessible to astronomical community from the NASA NSS Data Center. Send e-mail to: archives@nssdc.gfc.nasa.gov with the subject line: REQUEST OGLE ALL and put requested frame numbers (in the form MR00NNNN where NNNN stands for frame number according to OGLE notation), one per line, in the body of the message. Requested frames will be available using an "anonymous ftp" service from nssdc.gfc.nasa.gov 


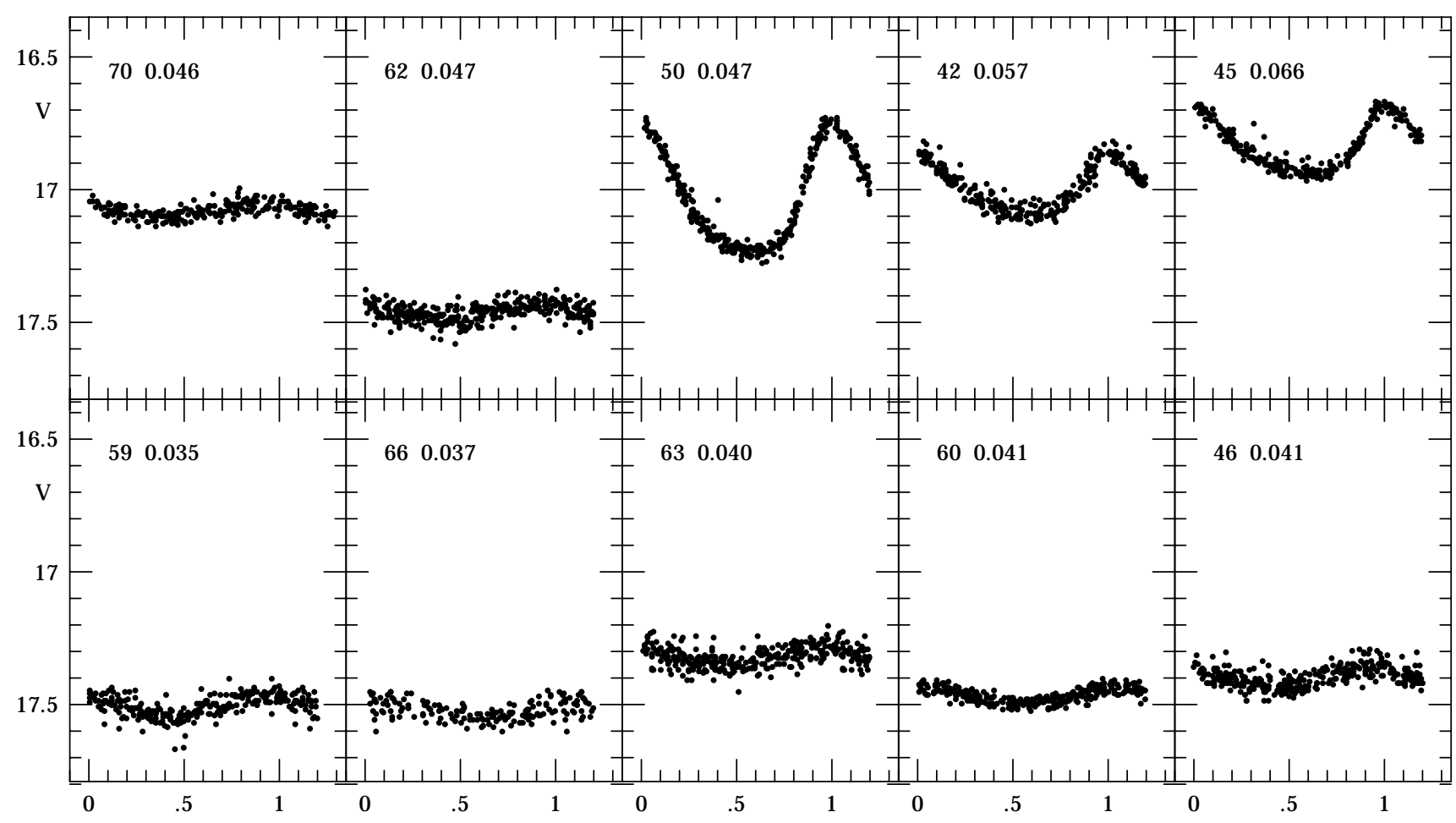

Fig. 2. Phased $V$ light curves for $10 \mathrm{SX}$ Phe stars identified in fields 5139D-F. Note the same height of all boxes. Variables are sorted according to their periods: inserted labels give names of variables and their periods in days

templates for fields 5139D, 5139E and 5139F, respectively. The transformation from rectangular to equatorial coordinates was derived based on positions of stars which could be matched with objects from the Guide Star Catalogue (Lasker et al. 1988). We identified 77, 106 and 82 GSC stars for fields 5139D, 5139E and 5139F, respectively. The adopted frame solutions reproduce equatorial coordinates of GSC stars with residuals rarely exceeding 0.5 arcsec.

Our sample of variables consist of 10 SX Phe stars and 19 certain or probable eclipsing binaries. Objects OGLEGC17 and OGLEGC20 were already reported in Paper I. These two stars are located in the overlapping areas of fields $5139 \mathrm{~A} / \mathrm{E}$ and $5139 \mathrm{C} / \mathrm{F}$. Variables OGLEGC42 = E39, OGLEGC50 = NJL220 and OGLEGC52 = Martin 78 were known before our survey (Jorgensen \& Hansen 1984; Martin 1938).

Table 5 lists basic characteristics of the light curves of 10 SX Phe star identified in fields 5139D-F. The mean $V$ magnitudes were calculated by numerically integrating the phased light curves after converting them into intensity scale. Photometric data for the remaining variables are given in Table 6 . To determine the most probable periods we used an aov statistic (Schwarzenberg-Czerny 1989, 1991). This statistic allows - in particular - reliable determination of periods for variables with non-sinusoidal light curves (eg. eclipsing variables of EA-type). Phased light

host in location shown in the return e-mail message from archives@nssdc.gsfc.nasa.gov curves of SX Phe stars are shown in Fig. 2 while Figs. 3 and 4 present light curves for the remaining variables.

Figure 5 shows location of variables with known color on the cluster color-magnitude diagram (CMD). For the SX Phe stars marked positions correspond to the intensity-averaged magnitudes. For the remaining variables we marked positions corresponding to magnitude at maximum light. The main sequence and red giant branch are marked with double lines in Fig. 5. The large width of these sequences is due to the well known spread of metallicity exhibited by stars in $\omega$ Cen (Woolley 1966).

All SX Phe stars as well as some eclipsing binaries are located on the cluster CMD among candidate blue stragglers. Four eclipsing binaries occupy positions at the base of the subgiant branch. Among them there are two systems with EA-type light curves: OGLEGC17 and OGLEGC51. The first system has been already reported in Paper I. The second one is a new discovery. The orbital period of OGLEGC51, $P=4.64$ day, connected with the shape of its light curve indicate that this binary is a detached system. The phase coverage at the primary minimum is poor and observed depth of the primary eclipse should be treated as a lower limit only. The variable was caught twice in the primary eclipse and twice in the secondary eclipse. This allowed to determine the period of OGLEGC51 with confidence.

Five variables occupy positions on the upper part of the main-sequence of the cluster. OGLEGC53 exhibit 

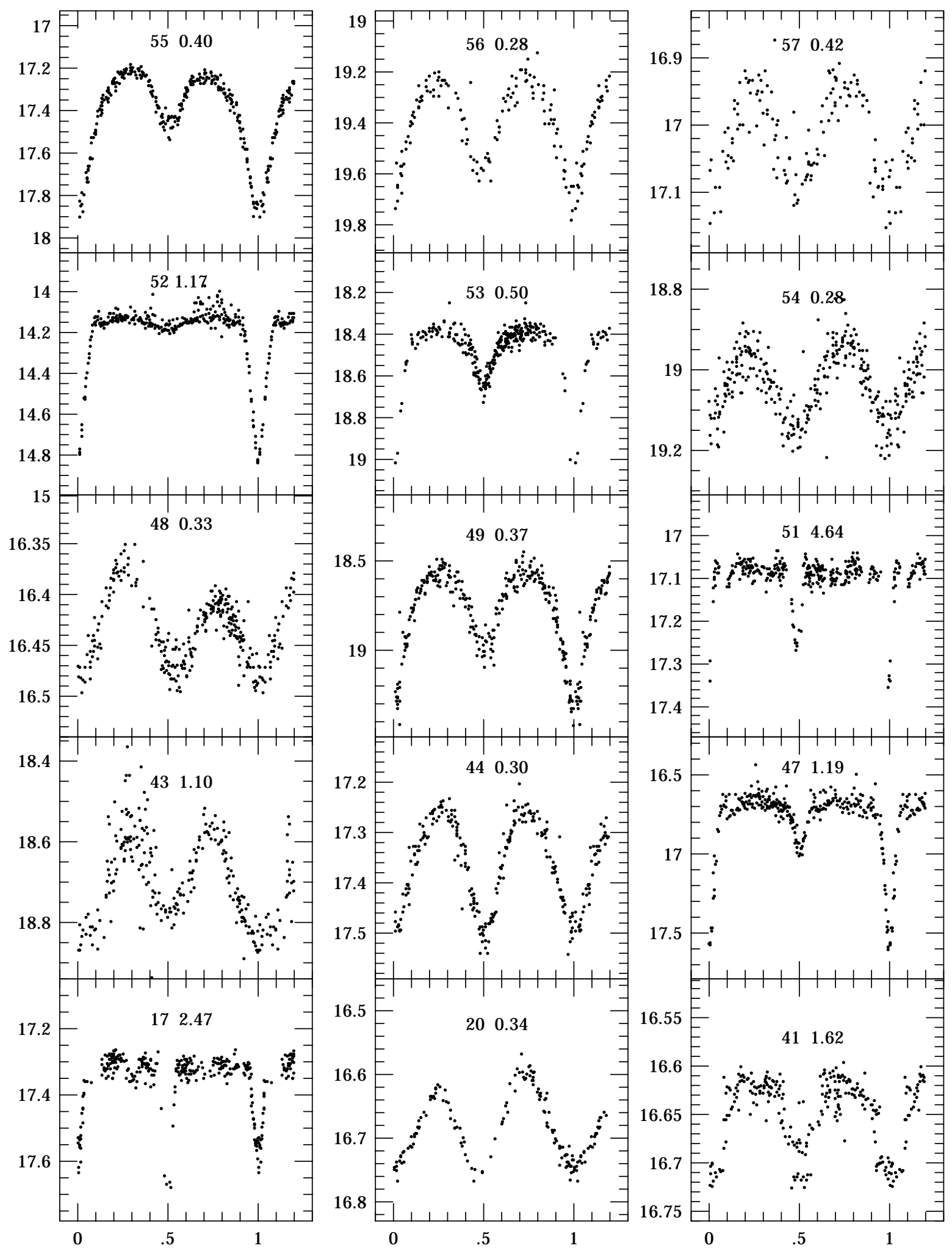

Fig. 3. Phased $V$ light curves for non-pulsating variables identified in fields 5139D-F. Inserted labels give names of variables and their periods 
Table 4. Rectangular and equatorial coordinates for variables identified in $\omega$ Cen. The $X$ and $Y$ coordinates give positions of variables on the template images (see text for details). The last column gives alternative names for two variables which were previously known. Variability of OGLEGC17 and OGLEGC20 was already reported in Paper I

\begin{tabular}{|c|c|c|c|c|c|c|}
\hline Name & $X$ & $Y$ & $\begin{array}{r}\mathrm{RA}(2000) \\
(\mathrm{h}: \mathrm{m}: \mathrm{s})\end{array}$ & $\begin{array}{r}\operatorname{Dec}(2000) \\
\text { (deg:':") }\end{array}$ & Field & $\begin{array}{l}\text { Other } \\
\text { Name }\end{array}$ \\
\hline OGLEGC17 & 113.69 & 1800.14 & $13: 27: 21.87$ & $-47: 23: 32.2$ & $5139 \mathrm{E}$ & \\
\hline OGLEGC20 & 99.82 & 1573.85 & $13: 27: 21.71$ & $-47: 37: 19.6$ & $5139 \mathrm{~F}$ & \\
\hline OGLEGC41 & 683.38 & 803.52 & $13: 25: 08.91$ & $-47: 30: 24.9$ & $5139 \mathrm{D}$ & \\
\hline OGLEGC42 & 551.31 & 1493.66 & $13: 25: 01.10$ & $-47: 25: 29.9$ & 5139D & E39 \\
\hline OGLEGC43 & 1002.56 & 1873.04 & $13: 25: 19.23$ & $-47: 22: 31.3$ & 5139D & \\
\hline OGLEGC44 & 1751.23 & 1593.30 & $13: 25: 52.34$ & $-47: 24: 07.9$ & 5139D & \\
\hline OGLEGC45 & 1770.24 & 1620.64 & $13: 25: 53.08$ & $-47: 23: 55.4$ & 5139D & \\
\hline OGLEGC46 & 341.21 & 975.77 & $13: 27: 34.05$ & $-47: 29: 23.4$ & $5139 \mathrm{E}$ & \\
\hline OGLEGC47 & 215.52 & 987.21 & $13: 27: 28.68$ & $-47: 29: 22.3$ & $5139 \mathrm{E}$ & \\
\hline OGLEGC48 & 248.03 & 1405.29 & $13: 27: 28.78$ & $-47: 26: 19.7$ & $5139 \mathrm{E}$ & \\
\hline OGLEGC49 & 448.23 & 1741.81 & $13: 27: 36.24$ & $-47: 23: 47.5$ & $5139 \mathrm{E}$ & \\
\hline OGLEGC50 & 780.64 & 596.16 & $13: 27: 53.92$ & $-47: 31: 54.4$ & $5139 \mathrm{E}$ & NJL220 \\
\hline OGLEGC51 & 596.32 & 901.86 & $13: 27: 45.13$ & $-47: 29: 47.6$ & $5139 \mathrm{E}$ & \\
\hline OGLEGC52 & 607.51 & 1403.16 & $13: 27: 44.06$ & $-47: 26: 09.7$ & $5139 \mathrm{E}$ & Martin 78 \\
\hline OGLEGC53 & 1497.66 & 999.54 & $13: 28: 23.28$ & $-47: 28: 37.0$ & $5139 \mathrm{E}$ & \\
\hline OGLEGC54 & 1761.09 & 167.23 & $13: 28: 37.19$ & $-47: 34: 29.0$ & $5139 \mathrm{E}$ & \\
\hline OGLEGC55 & 1742.23 & 1286.89 & $13: 28: 32.82$ & $-47: 26: 24.7$ & $5139 \mathrm{E}$ & \\
\hline OGLEGC56 & 1052.01 & 357.10 & $13: 28: 06.77$ & $-47: 45: 33.8$ & $5139 \mathrm{~F}$ & \\
\hline OGLEGC57 & 1141.94 & 1016.56 & 13:28:08.31 & $-47: 40: 45.2$ & $5139 \mathrm{~F}$ & \\
\hline OGLEGC58 & 584.86 & 1662.45 & $13: 25: 02.01$ & $-47: 24: 15.7$ & $5139 \mathrm{D}$ & \\
\hline OGLEGC59 & 1589.98 & 1281.47 & $13: 25: 46.37$ & $-47: 26: 28.3$ & 5139D & \\
\hline OGLEGC60 & 227.27 & 110.09 & $13: 27: 31.80$ & $-47: 35: 42.6$ & $5139 \mathrm{E}$ & \\
\hline OGLEGC61 & 228.86 & 1350.74 & $13: 27: 28.14$ & $-47: 26: 44.0$ & $5139 \mathrm{E}$ & \\
\hline OGLEGC62 & 246.23 & 1035.29 & $13: 27: 29.84$ & $-47: 29: 00.5$ & $5139 \mathrm{E}$ & \\
\hline OGLEGC63 & 81.06 & 1204.51 & $13: 27: 22.31$ & $-47: 27: 52.0$ & $5139 \mathrm{E}$ & \\
\hline OGLEGC64 & 233.51 & 1222.93 & $13: 27: 28.73$ & $-47: 27: 39.4$ & $5139 \mathrm{E}$ & \\
\hline OGLEGC65 & 1825.39 & 1621.01 & $13: 28: 35.29$ & $-47: 23: 57.3$ & $5139 \mathrm{E}$ & \\
\hline OGLEGC66 & 51.12 & 1774.18 & $13: 27: 18.97$ & $-47: 35: 54.4$ & $5139 \mathrm{~F}$ & \\
\hline OGLEGC67 & 610.93 & 1091.16 & $13: 25: 04.92$ & $-47: 28: 22.5$ & $5139 \mathrm{D}$ & \\
\hline OGLEGC68 & 1410.64 & 1412.39 & $13: 25: 38.23$ & $-47: 25: 37.5$ & 5139D & \\
\hline OGLEGC69 & 1956.06 & 832.21 & $13: 26: 03.58$ & $-47: 29: 30.6$ & 5139D & \\
\hline OGLEGC70 & 355.10 & 1525.39 & $13: 24: 52.62$ & $-47: 25: 22.2$ & $5139 \mathrm{D}$ & \\
\hline
\end{tabular}

Table 5. Light-curve parameters for SX Phe stars from fields $\mathrm{D}-\mathrm{F}$ in $\omega$ Cen. $A_{V}$ is the range of observed variations in the $V$ band. $(V-I)$ is the observed color at the maximum light. The period is given in days

\begin{tabular}{rrrrrr}
\hline Name & Period & $\langle V\rangle$ & $V_{\max }$ & $A_{V}$ & $(V-I)$ \\
\hline OGLEGC42 & 0.05740 & 17.00 & 16.86 & 0.23 & 0.51 \\
OGLEGC45 & 0.06560 & 16.84 & 16.69 & 0.24 & 0.57 \\
OGLEGC46 & 0.04080 & 17.40 & 17.36 & 0.08 & 0.58 \\
OGLEGC50 & 0.04718 & 17.04 & 16.75 & 0.48 & 0.49 \\
OGLEGC59 & 0.03495 & 17.51 & 17.46 & 0.10 & 0.53 \\
OGLEGC60 & 0.04063 & 17.47 & 17.43 & 0.06 & 0.63 \\
OGLEGC62 & 0.04662 & 17.46 & 17.43 & 0.06 & 0.67 \\
OGLEGC63 & 0.03997 & 17.32 & 17.28 & 0.08 & 0.50 \\
OGLEGC66 & 0.03749 & 17.53 & 17.49 & 0.07 & 0.52 \\
OGLEGC70 & 0.04626 & 17.08 & 17.05 & 0.05 & 0.56 \\
\hline
\end{tabular}

EB-type light curve and has period close to 0.50 day. It may be either a semidetached or nearly contact binary. OGLEGC49, OGLEGC54 and OGLEGC56 show light curves and periods typical for W UMa-type contact binaries. OGLEGC43 also shows EW-type light curve but at the same time its period, $P=1.10$ day, is unexpectedly long for a contact binary of late spectral type. The light curve of this star shows two distinct minima. Hence, we can rule out a possibility that OGLEGC43 is a pulsating variable with $P=0.55$ day located in the galactic halo behind the cluster.

Another interesting binary from our list is OGLEGC52. This star is located slightly above the horizontal branch of the cluster near the blue edge of the instability strip. Its light curve suggests a semi-detached configuration with a red component filling its Roche lobe. The secondary eclipse is shallow but the depth of the 

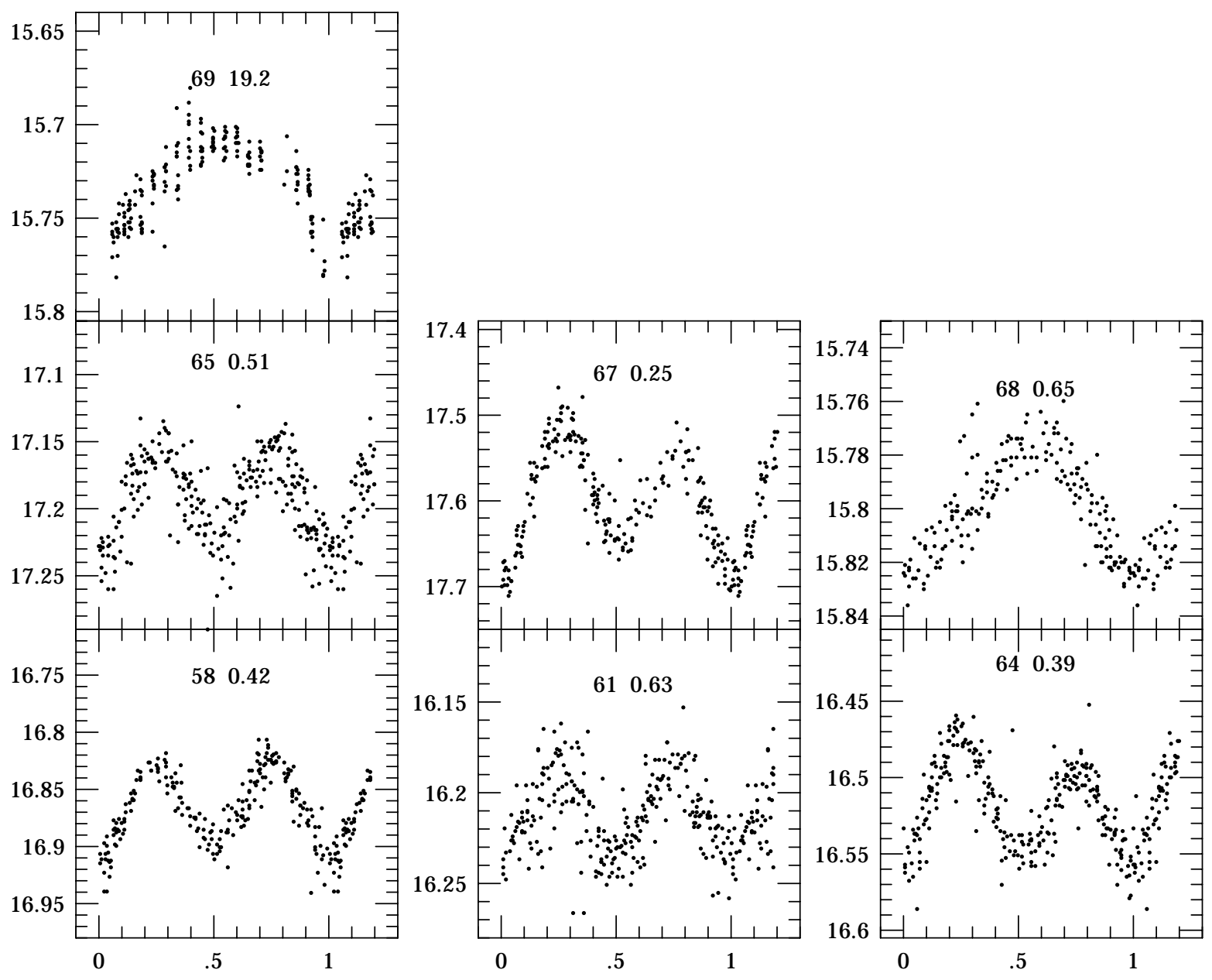

Fig. 4. Phased $V$ light curves for non-pulsating variables identified in fields 5139D-F. Inserted labels give names of variables and their periods

primary eclipse indicates comparable sizes of components of the system. OGLEGC52 may be composed of a horizontal branch star and a red sub-giant. On the other hand this star can be a field binary located in the cluster foreground. The out-of-eclipse part of the light curve does not show presence of any secondary periodicity. Hence, the blue component of OGLEGC52 is not a RR Lyr star. Some scatter visible in the light curve before primary eclipse is due to problems with photometring over-exposed images of the star.

We consider some of our period determinations, especially those for OGLEGC61 and OGLEGC65, as preliminary. For OGLEGC61 we obtained reasonable, lowamplitude light curves for two values of the orbital period: 0.631 and 0.479 day. For OGLEGC65 the possible periods are 0.512 and 0.408 day.

The periods of all identified SX Phe stars are rather firmly established. For variables of this type we calculated the power spectra using the program based on a CLEAN algorithm (Roberts et al. 1987). For all stars the derived power spectra show a very prominent peak at positions corresponding to the periods listed in Table 5 .

The variable OGLEGC41 is located about $0.3 \mathrm{mag}$ to the red of the subgiant branch of the cluster. We adopted for this star a period $P=1.62$ day but it cannot be ruled out that the real period is twice shorter. For $P=1.62$ day the likely reason of observed variability would be binary nature of OGLEGC41. This star could be either an eclipsing binary or an ellipsoidal variable. For $P=0.81$ day it could belong to BY Dra or FK Com type variables. The relatively red color of OGLEGC41 is consistent with the hypothesis that it belongs to spotted variables.

The contact binary OGLEGC67 is located more than $0.5 \mathrm{mag}$ to the red of the base of the subgiant branch on the cluster CMD. It is most probably a foreground star.

The low amplitude variable OGLEGC68 is located about 2 mag above cluster turnoff and 0.3 mag to the blue of the subgiant branch. We adopted for it a period of 0.65 day but the light curve can be phased also with a period of 1.3 day. For $P=0.65$ day the star could be a foreground spotted variable. For $P=1.3$ day it could 


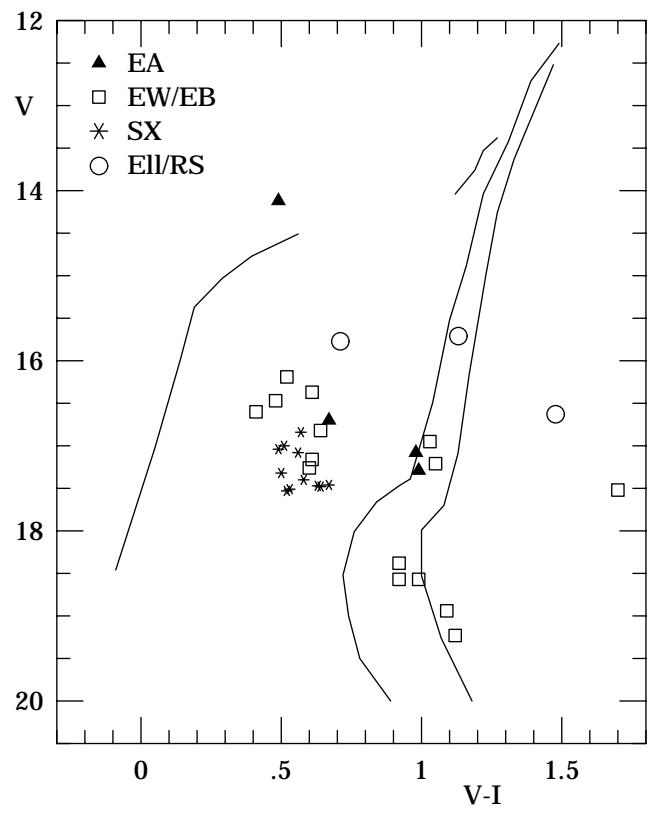

Fig. 5. The schematic CMD for $\omega$ Cen with positions of variables from fields D-F marked. The triangles represent EA-type eclipsing binaries, the squares EW/EB-type binaries, the asterisks SX Phe stars and the open circles probable ellipsoidal or spotted variables

be an ellipsoidal variable composed of subgiant and a star from an extended horizontal branch.

\subsection{Cluster membership of the variables}

The $\omega$ Cen cluster is located at an intermediate galactic latitude of $b=+15 \mathrm{deg}$. Therefore, one cannot assume that all variables listed in Table 4 are cluster members. Figure 6 shows the period versus absolute magnitude diagram for 10 SX Phe stars listed in Table 5. The standard relations for the $\mathrm{F}$-mode pulsators (solid line) and the $\mathrm{H}$ mode pulsators (dashed line) and for $[\mathrm{Fe} / \mathrm{H}]=-2.2$ (upper line) and $[\mathrm{Fe} / \mathrm{H}]=-0.7$ (lower line) are also shown. The calibration $P-L-[\mathrm{Fe} / \mathrm{H}]$ was taken from Nemec et al. (1994). We adopted for the cluster an apparent distance modulus $(m-M)_{V}=13.86$ while calculating absolute magnitudes of SX Phe stars. The assumed range of metallicities is based on results published by Brown \& Wallerstein (1993) and Vanture et al. (1994). The observed luminosities of reported SX Phe stars are consistent with the hypothesis that all of them are members of the cluster.

We have applied the absolute brightness calibration established by Rucinski (1995) to calculate $M_{V}$ for newly discovered contact binaries. Rucinski's calibration gives $M_{V}$ as a function of period, unreddened color $(V-I)_{0}$ and metallicity:

$$
\begin{aligned}
M_{V}^{\text {cal }}= & -4.43 \log (P)+3.63(V-I)_{0}-0.31-0.12 \\
& \times[\mathrm{Fe} / \mathrm{H}] .
\end{aligned}
$$

We adopted for all systems $[\mathrm{Fe} / \mathrm{H}]=-1.6$ what is the mean metallicity for the cluster stars. Figure 7 shows the period versus an apparent distance modulus diagram for contact binaries identified in fields 5139D-F of $\omega$ Cen. Specifically the following systems listed in Table 6 were considered to be "normal" W UMa-type binaries: OGLEGC20, 44, 48, 49, 54, 56, 57, 58, 61, 64, 65 and 67. An apparent distance modulus was calculated for each system as a difference between its $V$ magnitude and $M_{V}^{\text {cal }}$. An apparent distance modulus for $\omega$ Cen is estimated at $(m-M)_{V}=13.86$ (Nemec et al. 1994). The systems with mostly deviating values of $(m-M)_{V}$ are OGLEGC48, OGLEGC57 and OGLEGC67 (this system is not marked in Fig. 7). These binaries are probably a foreground variables. The remaining systems plotted in Fig. 7 are likely members of the cluster.

Clearly some additional data are needed to establish with more confidence which variables identified in our survey are members of $\omega$ Cen. We note that potential field interlopers should exhibit radial velocity differing significantly from the velocity observed for the cluster. Radial velocity of $\omega$ Cen is $+228 \mathrm{~km} / \mathrm{s}$ (e.g. Zinn 1985) which is a much higher value than velocities observed for field stars from the cluster region.

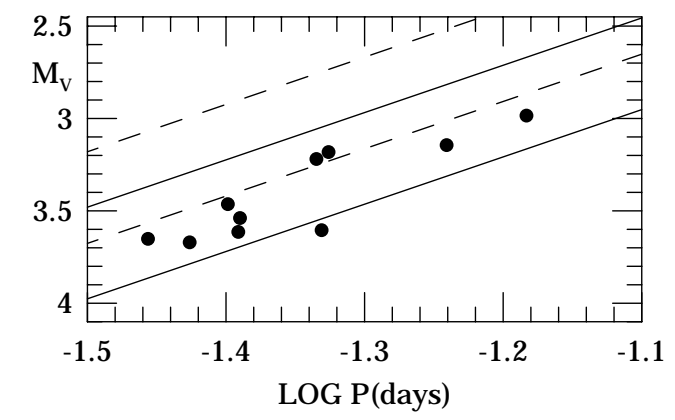

Fig. 6. Period vs. absolute magnitude diagram for SX Phe stars from the field of $\omega$ Cen

\section{The color-magnitude diagrams}

In Fig. 8 we show the $V$ vs. $V-I$ CMDs for fields 5139DF. For each field the photometry is based on a single pair of "long" V \& I exposures. The CMD's based on "short" exposures are presented in Fig. 9. The frames used for construction of CMD's shown if Figs. 8 and 9 are listed in Table 7. The presented CMDs were cleaned from stars of relatively poor photometry. In case of data plotted in Fig. 8 we removed $17 \%, 23 \%$ and $13 \%$ of stars with $V<18$ for fields 5139D, 5139E and 5139F, respectively.

The presented CMDs show the same features which have been already noted in Paper I. Several likely hot subdwarfs with $18.2<V<19.5$ and $(V-I) \approx 0.0$ can be noted. Also a numerous candidates for blue stragglers are 
Table 6. Light-curve parameters for eclipsing binaries discovered in fields D-F in $\omega$ Cen. $(V-I)$ is the observed color at the maximum light. $T_{0}$ is the time of minimum light

\begin{tabular}{rllllll}
\hline Name & Type & $\begin{array}{l}\text { Period } \\
\text { (days) }\end{array}$ & $V_{\max }$ & $V_{\min }$ & $V-I$ & $\begin{array}{l}T_{0} \text { HJD } \\
2449000+\end{array}$ \\
\hline OGLEGC17 & EA & 2.46655 & 17.29 & 17.67 & 0.99 & 818.7271 \\
OGLEGC20 & EW & 0.34183 & 16.60 & 16.75 & 0.41 & 818.8224 \\
OGLEGC41 & Ell/BY & $1.62 / 0.81$ & 16.62 & 16.71 & 1.48 & 516.3300 \\
OGLEGC43 & EW & 1.09644 & 18.57 & 18.84 & 0.99 & 516.4300 \\
OGLEGC44 & EW & 0.29631 & 17.26 & 17.49 & 0.60 & 515.7007 \\
OGLEGC47 & EA & 1.18870 & 16.70 & 17.58 & 0.67 & 819.2760 \\
OGLEGC48 & EW & 0.33188 & 16.37 & 16.48 & 0.61 & 818.7239 \\
OGLEGC49 & EB & 0.36627 & 18.57 & 19.30 & 0.92 & 818.5690 \\
OGLEGC51 & EA & 4.64042 & 17.08 & 17.34 & 0.98 & 820.7450 \\
OGLEGC52 & EA & 1.16807 & 14.12 & 14.83 & 0.49 & 818.8469 \\
OGLEGC53 & EB & 0.49519 & 18.38 & 19.00 & 0.92 & 818.8560 \\
OGLEGC54 & EW & 0.28377 & 18.94 & 19.18 & 1.09 & 818.7926 \\
OGLEGC55 & EB & 0.40389 & 17.21 & 17.85 & 1.05 & 818.7440 \\
OGLEGC56 & EW & 0.28118 & 19.23 & 19.73 & 1.12 & 818.7473 \\
OGLEGC57 & EW & 0.41783 & 16.95 & 17.09 & 1.03 & 818.7016 \\
OGLEGC58 & EW & 0.41577 & 16.82 & 16.92 & 0.64 & 515.6599 \\
OGLEGC61 & EW & 0.63093 & 16.19 & 16.23 & 0.52 & 818.9218 \\
OGLEGC64 & EW & 0.38505 & 16.47 & 16.56 & 0.48 & 818.8974 \\
OGLEGC65 & EW & 0.51224 & 17.16 & 17.23 & 0.61 & 818.8738 \\
OGLEGC67 & EW & 0.25064 & 17.52 & 17.69 & 1.70 & 515.7382 \\
OGLEGC68 & Ell/RS? & 0.65469 & 15.78 & 15.82 & 0.71 & 818.4927 \\
OGLEGC69 & RS? & 19.18 & 15.71 & 15.77 & 1.13 & 820.04 \\
\hline & & & & & &
\end{tabular}

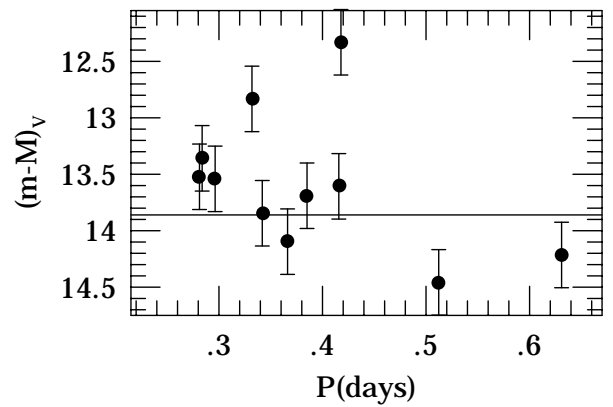

Fig. 7. Period vs. apparent distance modulus diagram for contact binaries from the field of $\omega$ Cen. A horizontal line at $(m-M)_{V}=13.86$ corresponds to distance modulus of the cluster. Error bars correspond to the formal uncertainty of absolute magnitudes derived using Rucinski's (1995) calibration

visible, especially in fields 5139D and 5139E. The apparent main sequence of field stars can be traced from the base of the subgiant branch of the cluster up to $V \approx 12.0$ which is the bright limiting magnitude of our photometry. This sequence is probably related to the so called "old disc" discussed recently by Jonch-Sorensen \& Knude (1994) and $\mathrm{Ng}$ et al. (1995).

All photometry presented in this section was submitted in tabular form to the editors of A\&A and is available in electronic form to all interested readers (see Appendix A).
Table 7. List of frames used for construction of CMDs shown in Figs. 8 and 9

\begin{tabular}{ccrrr}
\hline Frame & Field & $\begin{array}{r}T_{\text {exp }} \\
\text { sec }\end{array}$ & Filter & $\begin{array}{r}\text { FWHM } \\
\text { arcsec }\end{array}$ \\
\hline $\mathrm{mr} 10525$ & $5139 \mathrm{D}$ & 420 & $V$ & 1.01 \\
$\mathrm{mr} 10532$ & $5139 \mathrm{D}$ & 300 & $I$ & 0.97 \\
$\mathrm{mr} 10184$ & $5139 \mathrm{D}$ & 90 & $V$ & 0.94 \\
$\mathrm{mr} 10188$ & $5139 \mathrm{D}$ & 60 & $I$ & 1.14 \\
$\mathrm{mr} 10524$ & $5139 \mathrm{E}$ & 420 & $V$ & 1.01 \\
$\mathrm{mr} 10531$ & $5139 \mathrm{E}$ & 300 & $I$ & 0.99 \\
$\mathrm{mr} 10185$ & $5139 \mathrm{E}$ & 90 & $V$ & 0.98 \\
$\mathrm{mr} 10187$ & $5139 \mathrm{E}$ & 60 & $I$ & 0.99 \\
$\mathrm{mr} 11042$ & $5139 \mathrm{~F}$ & 420 & $V$ & 1.00 \\
$\mathrm{mr} 11447$ & $5139 \mathrm{~F}$ & 300 & $I$ & 1.01 \\
$\mathrm{mr} 10979$ & $5139 \mathrm{~F}$ & 90 & $V$ & 0.94 \\
$\mathrm{mr} 10998$ & $5139 \mathrm{~F}$ & 76 & $I$ & 1.07 \\
\hline
\end{tabular}

The potential users of this photometry should be aware about possibility of some systematic errors of the photometry. These errors are most likely to be significant for relatively faint stars. The CCD chip used for observations by the OGLE suffers from some nonlinearity. More details on this subject can be found in Paper I. 


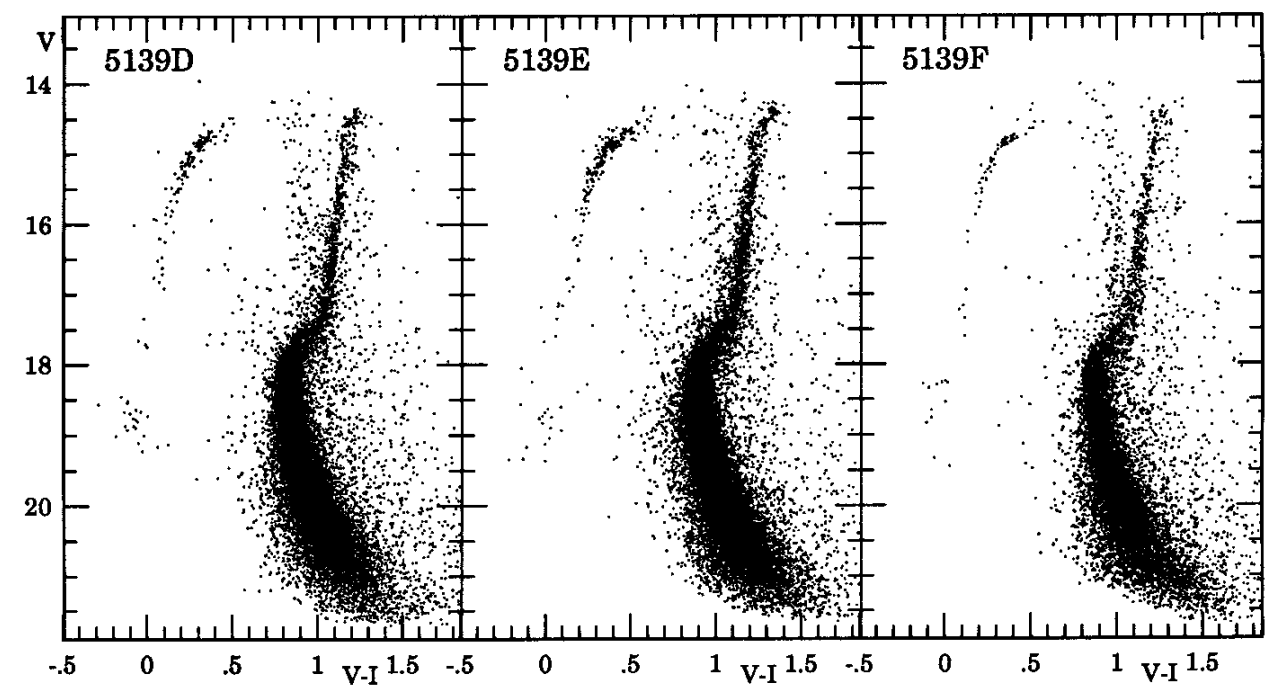

Fig. 8. The CMDs for fields 5139D (left), 5139E (center) and 5139F (right)

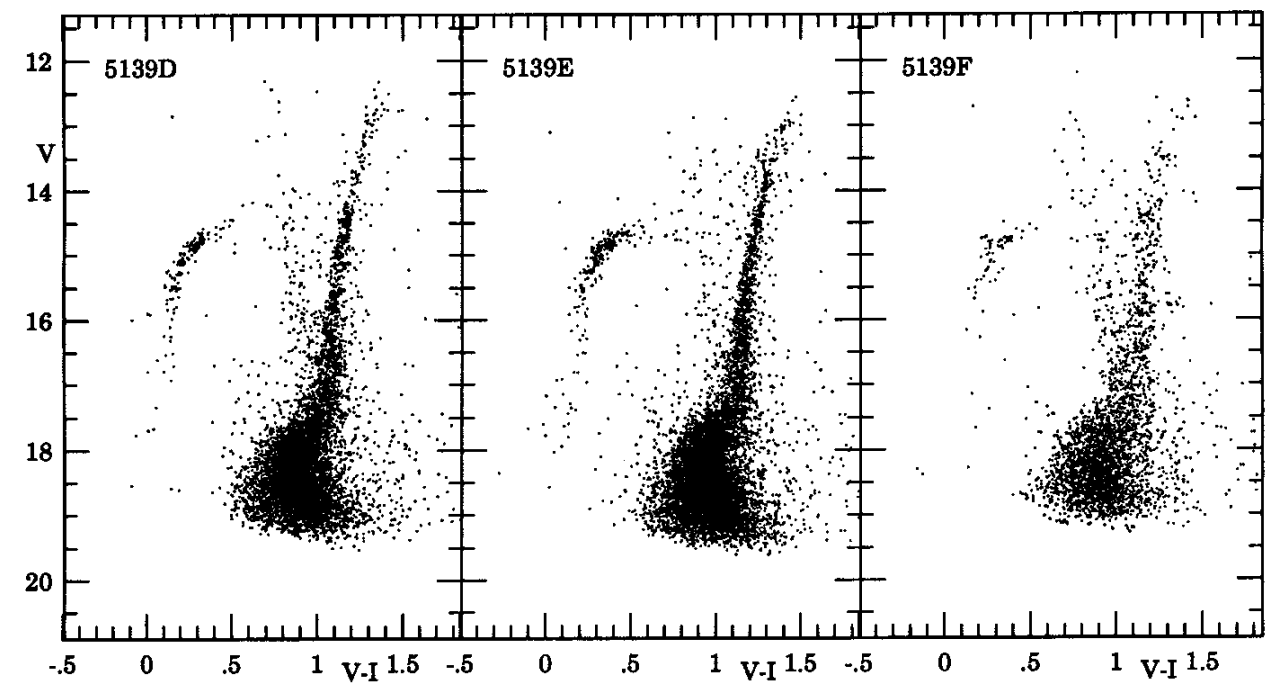

Fig. 9. The CMDs for fields 5139D (left), 5139E (center) and 5139F (right) based on shortly exposed frames

\section{Blue stragglers}

The CMD's of all monitored fields contain sizeable population of candidates for blue stragglers. Blue stragglers are known to be present in most well studied globular clusters (e.g. Ferraro et al. 1995). Several lines of evidence indicate that blue stragglers have higher masses than turnoff stars in their parent clusters. This supports hypothesis, originally proposed by McCrea (1964) that they are formed by merging of close binaries. In fact a few dozen photometric binary blue stragglers were discovered in globular clusters during recent years (see Mateo 1996 for a recent summary). These are mostly W UMa-type contact systems but some detached and semi-detached systems are also known. Another type of variable blue stragglers occurring in globulars are SX Phe stars. The fraction of variables seems to vary significantly from cluster to cluster. For example about $30 \%$ of blue stragglers in NGC 5053 are short-period variables (Nemec et al. 1995) while no single variable could be identified among 27 blue stragglers in NGC 6366 (Harris 1993). It is not clear for the moment how the relative frequency of variable blue stragglers correlates with such parameters as age and metallicity of a host cluster.

We used our data to estimate a relative frequency of contact binaries and SX Phe stars among $\omega$ Cen blue stragglers. It has to be noted at this point that the definition of blue stragglers is somehow flexible. It is unclear how to separate regions occupied by the turnoff stars and faint blue stragglers. For fields 5139A, 5139BC, 5139C and $5139 \mathrm{D}$ the following limits are adopted to define area 
occupied by blue stragglers: $16<V<18,0.30<V-I<$ 0.70 . For fields $5139 \mathrm{E}$ and $5139 \mathrm{~F}$ the corresponding limits are: $16<V<18,0.30<V-I<0.75$. The different definition of the red edge of the blue stragglers area reflects differences in the turnoff color observed for monitored fields. These differences may be possibly caused by the patchy reddening across the cluster face. The data presented in Fig. 8 and the data presented in Fig. 12 from Paper I were used to determine a number of blue stragglers in each of monitored fields. Note that presented CMD's are incomplete as we plotted only stars with relatively reliable photometry. The degree of incompleteness varies with changing crowding. Table 8 gives a number of selected blue stragglers candidates. Only objects whose light curves were analyzed for variability were retained in this statistic. The number of variable blue stragglers which were included in presented CMD's is also given in Table 8. All counted objects are blue stragglers according to adopted criteria on color and magnitude ${ }^{2}$. Note that some detected variable blue stragglers were dropped from the statistic. These were stars with formally poor photometry in the data sets used for plotting the CMD's. The fields 5139A and 5139C-F overlap with field 5139BC. Moreover, field $5139 \mathrm{~F}$ overlaps with fields $5130 \mathrm{E}$ and $5139 \mathrm{C}$. The statistics given in Table 8 applies to the non-overlapping parts of surveyed fields. The effective area of all fields are given in the second column of Table 8. We counted a total of 200 candidate blue stragglers. This number includes 24 SX Phe stars and 11 binaries (9 EW and 2 EB/EA systems). We showed that most of detected variables are very likely cluster members. The lower limits on the relative frequency of SX Phe stars and eclipsing binaries among cluster blue straggles are $(24 / 200)=0.12$ and $11 / 200=0.055$, respectively. It is difficult to asses precisely what fraction of candidate blue stragglers are field interlopers. However, we note that the surface density of possible blue stragglers is by a factor of $(92 / 10) \times 0.75=6.0$ higher for the central field 5139BC than for the outermost field 5139F. It may be concluded that most of selected blue stragglers are indeed members of the cluster. Our data indicate that $\omega$ Cen contains the largest number of blue stragglers among all well studied galactic globular clusters (e.g. Ferraro et al. 1995).

\section{Summary}

This section summarizes results presented above as well as those described in Paper I. We surveyed a total of six fields covering most of the central part of $\omega$ Cen and identified 70 periodic variables. This sample includes 6 previously known variables. RR Lyr stars are excluded

2 In fact we used for variables colors listed in Tables 5 and 6 (Tables 6 and 7 for fields studied in Paper I). The position marked on presented CMD's are based on a single pair V \& I of frames. Therefore they may be systematically in error in case of variable stars.
Table 8. Number of blue straggler candidates and number of variable blue stragglers in $6 \omega$ Cen fields. The second column gives relative area of a given field in units of area of the field 5139BC. $N(\mathrm{SX})$ and $N(\mathrm{ecl})$ denote numbers of SX Phe stars and eclipsing binaries, respectively. The last row summarizes data for all fields

\begin{tabular}{llrrr}
\hline Field & Area & $N(\mathrm{BS})$ & $N(\mathrm{SX})$ & $N(\mathrm{ecl})$ \\
\hline $5139 \mathrm{~A}$ & 0.94 & 25 & 7 & 1 \\
$5139 \mathrm{BC}$ & 1.0 & 92 & 6 & 5 \\
$5139 \mathrm{C}$ & 0.87 & 20 & 4 & 1 \\
$5139 \mathrm{D}$ & 0.92 & 26 & 4 & 1 \\
$5139 \mathrm{E}$ & 0.93 & 27 & 3 & 3 \\
$5139 \mathrm{~F}$ & 0.75 & 10 & 0 & 0 \\
$5139 \mathrm{~A}-\mathrm{F}$ & 5.41 & 200 & 24 & 11 \\
\hline
\end{tabular}

from these statistics - a separate paper devoted to them is in preparation. The most interesting result is identification of 3 detached eclipsing systems which are located at the base of the subgiant branch of the cluster. These are OGLEGC15, OGLEGC17 and OGLEGC51. Further observations of these systems can lead to direct determination of masses and radii of turnoff stars in $\omega$ Cen. An accurate determination of the cluster distance would be also obtained from such data. Another detached or semi-detached binaries identified in our survey are OGLEGC14 and OGLEGC52. The former one is a blue straggler (assuming it is a cluster member) while the latter is located slightly above the horizontal branch of the cluster.

We obtained light curves for 34 SX Phe stars. All of them are located among blue stragglers and are likely members of the cluster. 20 out of 34 of identified SX Phe stars shows variability with a full range not exceeding $0.1 \mathrm{mag}$ in the $V$-band. Detected SX Phe stars constitute about $12 \%$ of the blue straggler population of $\omega$ Cen.

Several contact or nearly-contact binaries were identified. Most of them are located among blue stragglers but some systems are present among upper main-sequence and turnoff stars. Most of identified contact binaries are likely members of the cluster.

Three low-amplitude variables with periods from the range 19-33 days were identified on the subgiant branch (OGLEGC22, OGLEGC35 and OGLEGC69). These stars belong most likely to the so called "spotted" variables. Another likely variable of this type is OGLEGC30 whose position on the CMD is unknown due to lack of information about its color.

Acknowledgements. This project was supported by NSF grants AST 92-16494 and AST-9313620 to Bohdan Paczynski and AST 92-16830 to George Preston. JK was supported by the Polish KBN grants 2P03D-008-08 and BST 501/17/95. MK, $\mathrm{MS}$ and AU were supported by the Polish KBN grant 2P03D029-08. 


\section{Appendix A}

Tables containing light curves of all variables discussed in this paper as well as tables with $V I$ photometry presented in Figs. 8 and 9 are published by A\&A at the centre de Données de Strasbourg, where they are available in electronic form via ftp 130.79.128.5 or http://cdsweb.ustrasbg.fr/abstract.html. Equatorial coordinates are given for all stars included in these tables. We have also submitted the $V$-filter "template" images of fields 5139D-F. These images can be used for identification of all variables discussed in this paper as well as for identification of all stars for which we provide VI photometry.

\section{References}

Brown J.A., Wallerstein G., 1993, AJ 106, 133

Ferraro F.R., Fusi Pecci F., Bellazzini M., 1995, A\&A 294, 80

Harris H.C., 1993, AJ 106, 604

Jonch-Sorensen H., Knude J., 1994, A\&A 288, 139

Jorgensen H., Hansen L., 1984, A\&A 133, 165

Kaluzny J., Kubiak M., Szymański M., Udalski A., Krzeminski W., Mateo M., 1996 A\&AS 120, 139 (Paper I)

Lasker B.M., et al., 1988, ApJS 68, 1

Martin W.C., 1938, Annalen van de Sterrewacht te Leiden 17,1

Mateo M.M., 1996, in: Binaries in Clusters, Milone E.F. (ed.).
ASP Conf. Ser. (in print)

McCrea W.H., 1964, MNRAS 128, 147

Nemec J.M., Linnell Nemec A.F., Lutz T.E., 1994, AJ 108, 222

Nemec J.M., Mateo M., Burke M., Olszewski E., 1995, AJ 110, 1186

Ng Y.K., Bertelli G., Bressan A., Chiosi C., Lub J., 1995, A\&A 295,66

Paczynski B., et al., 1995, IAU Symp. 169: Unsolved Problems of the Milky Way, Blitz L. (ed.) p. 133

Roberts D.H, Lehar J., Dreher J.W., 1987, AJ 93, 968

Rucinski S.M., 1995, PASP 107, 648

Schechter P., Mateo M., Saha A., 1993, PASP 105, 1342

Schwarzenberg-Czerny A., 1989, MNRAS 241, 153

Schwarzenberg-Czerny A., 1991, MNRAS 253, 198

Udalski A., Szymański M., Kałużny J., Kubiak M., Mateo M., 1992, Acta Astron. 42, 253

Udalski A., Szymański M., Kałużny J., Kubiak M., Mateo M., Krzemiński W., 1994, ApJ 426, L69

Udalski A., Szymański M., Kałużny J., Kubiak M., Mateo M., Krzemiński W., 1995, Acta Astron. 45, 237

Udalski A., Szymański M., Kałużny J., Kubiak M., Mateo M., Krzemiński W., Stanek K., 1996, Acta Astron. (in preparation)

Vanture A.D., Wallerstein G., Brown J.A., 1994, PASP 106, 835

Woolley R.v.dR., et al., 1966, R. Obs. Ann., No. 2

Zinn R., 1985, ApJ 293, 424 